eine gesetzliche Regelung am besten für Klarheit. Ein amtliches Interesse an einer allgemeinen Kenntnisnahme von Geodaten liegt zweifelsfrei dann vor, wenn eine Norm des öffentlichen Rechts den urheberrechtlichen Schutz von Geodaten begrenzt oder ausschließt. Eine vertragliche Regelung im Einzelfall würde deutlich größeren Aufwand verursachen und könnte die Gefahr nicht ausschließen, dass der jeweils zuständige Rechnungshof die Frage nach der Rechtsgrundlage aufwirft.

In diesem Zusammenhang ist zunächst das Informationsweiterverwendungsgesetz vom 13.12.2006 (IWG) zu betrachten. Im Wesentlichen trifft es die folgenden Regelungen: Dieses Gesetz gilt für die Weiterverwendung aller bei öffentlichen Stellen vorhandenen Informationen (§ 1 IWG). Weiterverwendung ist jede Nutzung von Informationen, die über die Erfüllung einer öffentlichen Aufgabe hinausgeht und in der Regel auf die Erzielung von Entgelt gerichtet ist. Die intellektuelle Wahrnehmung einer Information und die Verwertung des dadurch erlangten Wissens stellen regelmäßig keine Weiterverwendung dar (§ 2 Nr. 3 IWG). Jede Person ist bei der Entscheidung über die Weiterverwendung vorhandener Informationen öffentlicher Stellen, die diese zur Weiterverwendung bereitgestellt haben, gleich zu behandeln. Ein Anspruch auf Zugang zu Informationen wird durch dieses Gesetz jedoch nicht begründet ( $\$ 3$ IWG). Für Streitigkeiten nach diesem Gesetz ist der Verwaltungsrechtsweg eröffnet (§ 5 IWG).

Sodann war bis zum 15.5.2009 die Richtlinie 2007/2/EG des Europäischen Parlaments und des Rates vom 14.3.2007 zur Schaffung einer Geodateninfrastruktur in der Europäischen Gemeinschaft (INSPIRE) in nationales Recht umzusetzen. Der Bund ist dieser Verpflichtung mit Erlass des Gesetzes über den Zugang

${ }^{7}$ Auf dem Weg zum Open Government: Regimewechsel im Geodatenrecht, DVBI 2013, S. $1 \mathrm{ff}$. zu digitalen Geodaten - Geodatenzugangsgesetz (GeoZG) vom 10.2.2009 nachgekommen. Dieses Gesetz ist am 14.2.2009 in Kraft getreten.

Die INSPIRE-Richtlinie definiert, was "Geodaten" sind: alle Daten mit direktem oder indirektem Bezug zu einem bestimmten Standort oder geografischen Gebiet (Art. 3 Nr. 2 INSPIRE, § 3 Abs. 1 GeoZG).

Außerdem legt sie fest: Die Mitgliedstaaten schaffen und betreiben für Geodatensätze und Geodatendienste, für die gemäß dieser Richtlinie Metadaten erzeugt wurden, ein Netz, das folgende Dienste umfasst: Suchdienste, Darstellungsdienste, Download-Dienste, Transformationsdienste, Dienste zum Abrufen von Geodatendiensten. Diese Dienste müssen einfach zu nutzen, öffentlich verfügbar und über das Internet oder andere geeignete Telekommunikationsmittel zugänglich sein (Art. 11 Abs. 1 INSPIRE, § 6 GeoZG). Die Mitgliedstaaten sorgen dafür, dass Suchdienste und Darstellungsdienste der Öffentlichkeit kostenlos zur Verfügung gestellt werden (Art. 14 Abs. 1 INSPIRE).

Der Bund ist mit der am 16.11.2012 in Kraft getretenen Neufassung des Geodatenzugangsgesetzes über die Anforderungen der INSPIRE-Richtlinie hinausgegangen und hat einen großen Schritt in Richtung Open Government Data unternommen. Nach § 11 Abs. 2 GeoZG sind Geodaten und Metadaten über Geodatendienste für die kommerzielle und nicht kommerzielle Nutzung geldleistungsfrei zur Verfügung zu stellen, soweit durch besondere Rechtsvorschrift nichts anderes bestimmt ist oder vertragliche oder gesetzliche Rechte Dritter dem nicht entgegenstehen. Geodatenhaltende Stellen des Bundes stellen einander ihre Geodaten und Geodatendienste, einschließlich zugehöriger Metadaten, geldleistungsfrei zur Verfügung, soweit deren Nutzung zur Wahrnehmung öffentlicher Aufgaben erfolgt. Nach § 11 Abs. 3 GeoZG werden die
Einzelheiten zur Nutzung von Geodaten und Geodatendiensten in einer Rechtsverordnung nach § 14 GeoZG geregelt. In dieser Rechtsverordnung mit dem voraussichtlichen Namen "Geodatennutzungsverordnung" (GeoNutzV) werden insbesondere die Nutzungsbedingungen festgelegt werden. Der Abschluss von individuellen Nutzungsrechtsverträgen erübrigt sich insoweit.

Die geldleistungsfreie Nutzung der Geodaten des Bundes ist damit abschließend öffentlich-rechtlich geregelt. Das Urheberrechtsgesetz spielt insoweit keine Rolle mehr. Mario Martini und Matthias Damm bezeichnen diesen Schritt des Bundes als Regimewechsel im Geodatenrecht ${ }^{7}$.

$\S 11$ Abs. 2 GeoZG gibt die vom Geodatenzugangsgesetz erfassten Geodaten und Metadaten jedermann zur Verwertung frei. Eine analoge topografische Karte erfüllt allerdings nicht die Voraussetzungen dieser Bestimmung, da das Geodatenzugangsgesetz nur für Geodaten gilt, die in elektronischer Form vorliegen (§ 4 Abs. 1 Nr. 2 GeoZG).

Derzeit ist noch nicht absehbar, ob die Länder dem Beispiel des Bundes folgen werden. Baden-Württemberg hat Teile seiner Geobasisdaten durch Presseerklärung des Ministers für Ländlichen Raum und Verbraucherschutz Alexander Bonde vom 17.1.2013 für offen erklärt und sie unter die Lizenz CC BY 3.0 gestellt. Unter anderem bietet Baden-

Württemberg den Geodatendienst "Maps4BW" geldleistungsfrei und ohne Nutzungsbeschränkung an. Einzige Bedingung ist die Quellenangabe "LGL, www.Igl-bw.de". Eine Presseerklärung hat natürlich keine normative Kraft wie der Erlass eines Gesetzes oder einer Rechtsverordnung. Stuft man die Presseerklärung als schlicht hoheitliche Handlung ein, dann könnte man die davon betroffenen Geodaten als durch hoheitlichen Organisationsakt für offen erklärte Daten bezeichnen. Auch die Lizenz CC BY 3.0 könnte dadurch für die unter diese Lizenz gestellten Daten der Vermessungsverwaltung BadenWürttemberg öffentlich-rechtlichen Charakter bekommen. Allerdings ist diese Sichtweise rechtlich nicht zwingend. Genauso könnte man die Auffassung vertreten, ein öffentlichrechtliches Nutzungsverhältnis liege nicht vor und bei der Nutzung offener Daten der Vermessungsverwaltung in Baden-Württemberg komme es nur zu einem zivilrechtlichen Vertrag zwischen dem Land und dem Nutzer. Dann wäre auch das Urheberrechtsgesetz weiterhin im Grundsatz zu beachten.

Die Klärung dieser Frage dürfte der weiteren Entwicklung des Projekts „Open Government Data” vorbehalten bleiben.

\section{Über den Verfasser:}

Ltd. Regierungsdirektor Dietrich Diez ist Referatsleiter im Landesamt für Geoinformation und Landentwicklung Baden-Württemberg und Leiter der DGfKKommission Recht und Geodaten. E-Mail: dietrich.diez@|gl.bwl.de

\section{Ereignisse}

\section{- Eröffnung der Ausstellung „Den Seuchen auf der Spur" am 19.9.2013 in Hannover}

\section{Bericht}

In Anwesenheit von 130 geladenen Gästen hat der Präsident des Niedersächsischen Landtages Hermann Dinkla am 19. September 2013 die Ausstellung „Den Seuchen auf der Spur - 200 Jahre Infektionskrankheiten im Kartenbild" offiziell eröffnet. Die Ausstellung zeigt Karten zum Thema "Infektionskrankheiten" aus dem 19., 20. und 21. Jahrhundert, darunter auch Kartenoriginale aus den Beständen der Staatsbibliothek zu Berlin, und wurde gemeinsam 


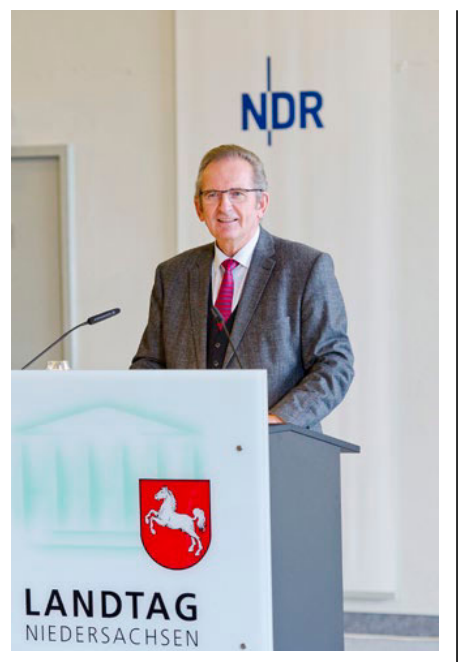

Der Präsident des Niedersächsischen Landtages Hermann Dinkla eröffnet die Ausstellung.

vom Niedersächsischen Landesgesundheitsamt, der Deutschen Gesellschaft für Kartographie und der Staatsbibliothek zu Berlin - Preußischer Kulturbesitz zum 60. Deutschen Kartographentag und der INTERGEO in Hannover ausgerichtet.

In seiner Eröffnungsrede hat der Landtagspräsident auf die Bedeutung des Themas "Seuchen" in der gesamten Menschheitsgeschichte hingewiesen. Dabei betonte er, dass Infektionskrankheiten in den Industrieländern zwar viel von ihrem Schrecken verloren haben, uns Krankheitsausbrüche wie EHEC/HUS 2011 aber vor Augen führen, dass wir trotz der Fortschritte im Kampf gegen Viren und Bakterien vor neuen Infektionsausbrüchen nicht geschützt sind. Karten, so der Landtagspräsident, waren und sind ein zentrales Werkzeug für die Ermittlungsarbeit und die Information der Öffentlichkeit.

Im Anschluss wies Matthias Pulz, Präsident des Niedersächsischen Landesgesundheitsamtes, in seinem Grußwort auf die zentrale Rolle hin, die Karten in der Arbeit des Landesgesundheitsamtes einnehmen, sei es für die Trinkwasserberichterstattung, das Niedersächsische Epidemiologische Krebsregister oder die Information der Öffentlichkeit über die Badegewässerqualität in Niedersachsen. Mit Blick auf die Ausstellung betonte er, dass Karten komplexe Aspekte eines Ausbruchsgeschehens in anschaulicher und verständlicher Form hervorheben und Ausgangspunkt für weitergehende Fragestellungen sein können.

Einen kurzweiligen Einblick in die lange Tradition der Verbindung zwischen Geographie und Medizin gab Thomas Kistemann (Universität Bonn, Institut für Hygiene und öffentliche Gesundheit) in seinem Fachvortrag mit dem Thema "Medizinische Karten - ein geographisches Werkzeug zur Erkennung, Kontrolle und Prävention von Infektionshäufungen ". Anhand von Beispielen stellte er u.a. dar, dass im 17./18 Jh. über den Zusammenhang zwischen den Umweltbedingungen und der Gesundheit berichtet wurde. So wurde mit Blick auf die kolonialzeitlichen Auswanderungen von zeitgenössischen Autoren das gestörte Wohlbefinden des Menschen in fernen Ländern mit dem von Pflanzen verglichen, die, an andere Orte verpflanzt, unter anderen Umweltbedingungen (Boden, Klima etc.) in ihrer Entwicklung beeinträchtigt werden.

Mit einem Zitat aus dem Roman "Das Buch, in dem die Welt verschwand" von Wolfram Fleischhauer begann Wolfgang Crom, Leiter der Kartenabteilung der Staatsbibliothek zu Berlin, seine Einführung in die Ausstellung. In dem Zitat wird am Beispiel der Karte des Londoner Arztes John Snow aus der Mitte des 19. Jh. die Bedeutung von Karten für die kriminalistische Aufklärungsarbeit herausgestellt. Daran anknüpfend zeigte Wolfgang Crom anhand ausgewählter Beispiele, die auch in der Ausstellung zu sehen waren, die Entwicklung von Infektionskarten in den vergangenen 200 Jahren auf.

Beim anschließenden Empfang hatten die Gäste der Eröffnungsveranstaltung die Gelegenheit, an einem Rundgang durch die Ausstellung teilzunehmen. Holger Scharlach, Kurator der Ausstellung, stellte im Rahmen des Rundgangs sieben Kartenbeispiele aus dem 19., 20. und 21. Jh. mit ergänzenden Materialien vor.
Die Ausstellung wird 2013 im Robert Koch-Institut Berlin und im Wissenschaftszentrum Bonn gezeigt Weitere Informationen auf der Internetseite: http://www.den-seuchenauf-der-spur.nlga.niedersachsen.de

Holger Scharlach, Hannover

\section{Hochschul- nachrichten}

\section{- Die Geo-Studiengänge an der Hochschule Karlsruhe - Technik und Wirtschaft: \\ Geoinformationsmanage- ment - Geodäsie und Navigation - Geomatics}

Mit dem Wintersemester 2012/13 wurden die Geo-Studiengänge an der Hochschule Karlsruhe neu strukturiert. Der Bachelorstudiengang Vermessung und Geomatik wurde mit einer neuen Vertiefungsrichtung "Navigation" erweitert und in "Geodäsie und Navigation" umbenannt. Die Kartographie wurde in den 2009 eingeführten, sehr erfolgreichen Bachelor-Studiengang Geoinformationsmanagement als Vertiefungsrichtung integriert. Darauf aufbauend werden wie bisher der internationale Masterstudiengang Geomatics und seine dreisemestrige deutsche Variante Geomatik angeboten. Die Studiengänge sind Bestandteil der neu gegründeten Fakultät für Informationsmanagement und Medien der Hochschule Karlsruhe, deren weitere Studiengänge Verkehrssystemmanagement, KulturMediaTechnologie sowie Kommunikation und Medienmanagement sich in anderer Weise dem Raumbezug, der Informationsverarbeitung und der Präsentation von Informationen widmen.

Der Bachelorstudiengang Geoinformationsmanagement bietet eine effiziente Kombination aus Geodatenaufbereitung, Analysen zur Ableitung neuer Informationen und graphischer Gestaltung. Im Grundstudium, den ersten beiden Semestern, werden Kenntnisse über die Erfassung, Qualität und Anwendbarkeit von Geodaten vermittelt. Studieninhalte wie Geodatenerfassung, Geographie, Mathematik, Informatik, Kartographie und Visualisierung vermitteln die Grundlagen für die aufbauenden, am gesellschaftlichen und wirtschaftlichen Bedarf orientierten Vertiefungsrichtungen, die ab dem 3. Fachsemester durchgeführt werden: Geomarketing, Kartographie \& Geomedien sowie Umwelt. Der gemeinsame Schwerpunkt im Hauptstudium liegt auf den Geographischen Informationssystemen (GIS), der wichtigen Basistechnologie, welche die digitale Schnittstelle zwischen Input, Analyse und Output der raumbezogenen Informationen bildet.

Betriebswirtschaft, Marketing und verschiedene Geomarketing-Anwendungen bereiten die Studierenden der Vertiefung Geomarketing auf praktische Aufgaben wie z.B. Standortplanungen, Marktanalysen oder Händlernetzentwicklung vor. Die graphische Aufbereitung von raumbezogenen Informationen in Karten und elektronischen Medien, Karten- und Mediendesign, Kartenredaktion, Interaktive und Echtzeit-Kartographie sind Schwerpunkte der Vertiefung Kartographie \& Geomedien. Umweltmonitoring, Fernerkundung, Raumplanung, Risiko- und Katastrophenmanagement sowie GIS-Anwendungen im Umweltbereich sind Themen der Vertiefungsrichtung Umwelt, die sich mit der Konzeption und dem Management von UmweltmonitoringSystemen befasst.

Neben den Vertiefungsrichtungen werden für alle Studierenden des Geoinformationsmanagements Lehrveranstaltungen zu Informationssystemen und Datenbanken, GIS-Programmierung, Geodateninfrastrukturen und WebServices sowie 3D-Visualisierung angeboten.

Durch die Integration des Bachelor-Studiengangs "Kartographie und Geomatik" sind die 30 Studien- 